\title{
Multidimensional Longwave Forcing of Boundary Layer Cloud Systems
}

\author{
David B. Mechem, ${ }^{*}+$ Yefim L. Kogan, ${ }^{+}$Mikhail Ovtchinnikov,\# Anthony B. Davis, \\ K. Franklin Evans, \& and Robert G. ElLINGSON** \\ * Department of Geography, University of Kansas, Lawrence, Kansas \\ + Cooperative Institute for Mesoscale Meteorological Studies, University of Oklahoma, Norman, Oklahoma \\ \# Pacific Northwest National Laboratory, Richland, Washington \\ @ Los Alamos National Laboratory, Los Alamos, New Mexico \\ \& University of Colorado, Boulder, Colorado \\ ** The Florida State University, Tallahassee, Florida
}

(Manuscript received 11 January 2008, in final form 24 April 2008)

\begin{abstract}
The importance of multidimensional (MD) longwave radiative effects on cloud dynamics is evaluated in an eddy-resolving model (ERM) - the two-dimensional analog to large-eddy simulation (LES) - framework employing multidimensional radiative transfer [Spherical Harmonics Discrete Ordinate Method (SHDOM)]. Simulations are performed for a case of unbroken, marine boundary layer stratocumulus and a broken field of trade cumulus. "Snapshot" calculations of MD and independent pixel approximation (IPA; 1D) radiative transfer applied to simulated cloud fields show that the total radiative forcing changes only slightly, although the MD effects significantly modify the spatial structure of the radiative forcing. Simulations of each cloud type employing MD and IPA radiative transfer, however, differ little. For the solid cloud case, relative to using IPA, the MD simulation exhibits a slight reduction in entrainment rate and boundary layer total kinetic energy (TKE) relative to the IPA simulation. This reduction is consistent with both the slight decrease in net radiative forcing and a negative correlation between local vertical velocity and radiative forcing, which implies a damping of boundary layer eddies. Snapshot calculations of the broken cloud case suggest a slight increase in radiative cooling, although few systematic differences are noted in the interactive simulations. This result is attributed to the fact that radiative cooling is a relatively minor contribution to the total energetics. For the cloud systems in this study, the use of IPA longwave radiative transfer is sufficiently accurate to capture the dynamical behavior of boundary layer clouds. Further investigations are required to generalize this conclusion for other cloud types and longer time integrations.
\end{abstract}

\section{Introduction}

The complex feedbacks between radiation and clouds are poorly understood and remain one of the greatest uncertainties in simulations of global climate change scenarios (Houghton et al. 2001). Accurately representing the interactions between solar and thermal radiation and specified cloud fields is a first step in understanding this complicated cloud-radiation feedback. Numerical models ranging from global climate

Corresponding author address: David B. Mechem, Department of Geography, University of Kansas, 1475 Jayhawk Blvd., 213 Lindley Hall, Lawrence, KS 66045-7613.

E-mail:dmechem@ku.edu models down to large-eddy simulation (LES) models nearly universally employ one-dimensional treatments of radiative transfer (RT), typically implemented as a two- or four-stream approximation to the radiative transfer equation. One-dimensional RT (1DRT), also referred to here as the independent pixel approximation (IPA), ${ }^{1}$ is computationally attractive relative to Monte Carlo methods or solving the full radiative transfer equation. However, IPA RT neglects horizontal ra-

\footnotetext{
${ }^{1}$ IPA is frequently confused with the plane-parallel approximation. IPA RT is based on the full vertical cloud structure for each pixel (or model column, in our case) but neglects horizontal radiation transport. Plane-parallel RT employs spatially averaged cloud properties, which leads to bias in certain radiative quantities such as albedo (Cahalan et al. 1994a).
} 
diation transport ${ }^{2}$ and associated effects such as cloud shadowing and radiative cooling of cloud lateral boundaries, which may be important for situations of complex cloud geometry and internal cloud structure. The Intercomparison of 3D Radiation Codes project (I3RC; Cahalan et al. 2005) has demonstrated that atmospheric radiative transfer is fundamentally $3 \mathrm{D}$, and that employing IPA heating rates can, for example, introduce systematic bias, leading to significant errors in domainaverage shortwave heating rates.

Evaluating multidimensional (MD) radiative effects in the shortwave has been a topic of investigation for over 30 yr. Early studies employed Monte Carlo radiative transfer to idealized geometrical configurations of cuboidal clouds. Applying this approach, Welch and Wielicki (1984) found that the plane-parallel assumption introduces significant error $\left(>10 \mathrm{~W} \mathrm{~m}^{-2}\right)$ in domain-mean calculations of shortwave flux for cloud fractions between $10 \%$ and $90 \%$. Harshvardhan and Thomas (1984) analyzed a two-dimensional configuration of infinitely long clouds taken to be representative of cloud sheets and developed a simple correction to the plane-parallel fluxes. More recent studies (e.g., Davis et al. 1997; Zuidema and Evans 1998; Di Giuseppe and Tompkins 2003) have explored the MD effect on albedo for boundary layer stratocumulus cloud fields. These studies typically calculate MD and IPA radiative transfer and subsequently evaluate the "plane-parallel albedo bias" (Cahalan et al. 1994a), an important domain-average quantity for assessing the fidelity of the shortwave radiation budget and its impact on the global energy balance. Closer to our present concern is what Cahalan et al. (1994b) call the IPA bias, namely the MD-IPA difference in mean or local quantities, which is controlled by the degree and kind of spatial heterogeneity in the cloud field, with broken cloud systems exhibiting greater bias than solid cloud fields. The above studies have shown that, for domainaverage fluxes in overcast cloud fields, the shortwave IPA bias is small for near-zenith illumination and insignificant for more typical incidence angles (compared to other uncertainties in the radiation budget). They also show that local MD-IPA differences can be very large in absolute value.

Studies exploring MD effects in the longwave are less common and have generally addressed the effects of cloud geometry or scattering on radiative fluxes. Using

\footnotetext{
${ }^{2}$ This mesoscopic process is often described as "photon transport." This is a misnomer because the particle-like nature of light only manifests itself at the microscopic level in interaction with matter (e.g., photon-counting detectors or photons emitted in a laser pulse); see Mishchenko (2008) and references therein.
}

a $3 \mathrm{D}$ version of the two-stream approximation, as well as Monte Carlo simulations, Harshvardhan and Weinman (1982) found that cloud fields of cumulus with cloud fractions of 0.3 could potentially exhibit an MD cooling rate up to 2-3 times that obtained from IPA radiative transfer. Ellingson (1982) derived analytical expressions representing an effective cloud fraction for idealized right circular cylinders. This effective cloud fraction represents the fraction of flat plates that would produce the same flux as the MD flux interacting with the actual cloud cover. Recognizing that convective clouds are frequently shaped like truncated cones, Killen and Ellingson (1994) derived expressions of effective cloud fraction for more complicated cloud geometries and found that although broken clouds do increase the effective cloud fraction, the effect is not as pronounced as in previous studies that employed simple cuboid geometries. Results from cuboidal cloud geometries were extended in Masunaga and Nakajima (2001) by relaxing the blackbody assumption and addressing how optical thickness influences the effective cloud fraction. Benassi et al. (2004) further extended these studies by using realistic cloud structures, broken or not, that were generated with a data-driven stochastic cloud model to compute the MD thermal radiative transfer. Scattering effects in the longwave have traditionally been thought to play only a minor role compared to absorption, and in a study of the relative contributions of cloud geometry and scattering Takara and Ellingson (1996) concluded that MD effects of cloud geometry indeed dominate over scattering. For a more detailed survey, we refer the reader to Ellingson and Takara (2005).

Past studies of MD effects were predominantly concerned with radiances and fluxes. MD effects in the shortwave alter the surface flux and heating rate, over time influencing cloud dynamics through changing the boundary layer equivalent potential temperature $\theta_{e}$, which in a very crude way may be thought to be proportional to convective intensity. Perhaps, too, the emergence of inhomogeneity in low-level $\theta_{e}$ arising from MD effects may also influence convective dynamics.

Instead of these predominantly indirect influences of MD radiative transfer (MDRT) on cloud dynamics, we are concerned with identifying direct impacts of MD effects on the cloud dynamics themselves. As such, we choose to focus on cloud types for which radiative forcing contributes significantly to the system energetics. For boundary layer stratocumulus, cloud top longwave radiational cooling is most frequently the primary engine driving negatively buoyant downdrafts and hence promoting the overall boundary layer circulation. Al- 
though stratocumulus cloud fields are to first order plane-parallel, the dynamics are highly dependent on the direct radiative forcing of the cloud, so it seems plausible that even small differences in the radiative forcing might influence cloud evolution. Evaporative feedback (buoyancy reversal; Siems et al. 1990; Moeng 2000) or drizzle (Stevens et al. 1998) may promote thinning or breakup of the cloud deck, which may serve to enhance or amplify MD effects. Radiative forcing is even more important for altocumulus clouds because they are radiatively cooled at the top, heated at the bottom, and are unaffected by the surface forcing (Liu and Krueger 1998). However, here we restrict our study to boundary layer clouds.

The importance of the radiative forcing contribution in isolated, shallow cumulus is less clear, although the small cloud fraction suggests that MD cooling of cloud lateral boundaries may play a role in the cloud dynamics. The irony of cloud systems with small cloud fraction, either broken stratocumulus or shallow cumulus, is that they tend to be more surface-driven from high $\theta_{e}$ parcels in the boundary layer, rather than being driven by radiative effects.

Most previous MDRT studies have been centrally concerned with how the cloud spatial structure modulates the radiative characteristics of a cloud system and do not address the direct influence of MD effects on the cloud dynamics. For example, how does using a full MD radiative forcing relative to $1 \mathrm{D}$ forcing affect cloud system evolution as measured by such quantities as buoyancy flux or entrainment? Guan et al. (1995) demonstrate that longwave MDRT can produce different stratocumulus cloud top cooling rates depending on whether the cloud top is flat or undulating. Their results suggest an interactive feedback between MDRT and cloud dynamics, although their experimental framework is not able to address the ultimate effect of such a feedback. Guan et al. (1997) show that longwave cooling on the sides of a small, slab-symmetric cumulus strengthens the cumulus downdraft and promotes new development near the cloud base. The authors include MDRT effects in their simulation but do not isolate the forcing arising from horizontal radiation flow from the total radiative forcing. One other notable work on interactive MD forcing of a cloud model is that of Koračin et al. (1998), in which the authors interactively couple Monte Carlo radiative transfer in the shortwave with a two-dimensional cloud model. They too, however, do not evaluate the overall sensitivity to radiative processes, nor do they isolate the MD effects.

Applying incorrect radiative forcing, either in magnitude or in distribution, has the potential to bias cloud system evolution. Boundary layer stratocumulus is the most obvious example of a cloud system predominantly driven by radiative processes, namely longwave cooling at cloud top. At first glance, 1DRT seems reasonable for clouds like stratocumulus that are to a great extent horizontally uniform. However, undulations in cloud top can result in radiative forcing different from the horizontally uniform value that 1DRT produces, and this can feed back into the dynamical fields. The historical stratocumulus literature is filled with discussion as to exactly where the radiative cooling should be placed and the consequences of applying it inappropriately (although much of this discussion takes place in the context of mixed layer models).

The present work seeks to evaluate the magnitude of the interactive nature of MD radiative transport on cloud dynamics. We believe our results not only shed light on the direct impact of MDRT on the cloud system structure and dynamics but also contain implications for the overall nature of radiative-dynamic interactions in clouds.

\section{Methodology}

We apply the multidimensional radiative transfer scheme of Evans (1998) - the Spherical Harmonics Discrete Ordinate Method (SHDOM) - to cloud fields obtained by an eddy-resolving model (ERM), which is the two-dimensional analog to LES, to produce longwave MD and IPA fluxes and heating rates. The independent pixel mode of SHDOM is used to obtain the IPA quantities. We employ two methods: first, a "snapshot" approach, in which the heating rate difference fields $\left(Q_{\mathrm{md}}-Q_{\text {ipa }}\right)$ are calculated using the same cloud fields. The difference field $Q_{\mathrm{md}}-Q_{\text {ipa }}$ is then interpreted in the context of ERM dynamic fields to infer feedbacks onto the cloud-topped boundary layer dynamics and cloud field structure relative to IPA forcing. We extracted all the snapshots from the ERM simulations using coupled MD radiative transfer. Second, we apply a fully interactive approach in which we couple SHDOM to an eddy-resolving model to address the interactive and evolutionary behavior of the MD-IPA bias and to quantify the ultimate importance of horizontal radiation transport for cloud system evolution.

\section{a. Instantaneous snapshot RT calculations}

The Cooperative Institute of Mesoscale Meteorological Studies (CIMMS) LES (Kogan et al. 1995; Khairoutdinov and Kogan 1999), run as a two-dimensional eddy-resolving model, supplies the cloud field liquid water content (LWC) data for the radiative transfer calculation. Cloud optical properties first are calcu- 
lated, and then SHDOM employs the correlated- $k$ distribution of Fu and Liou (1992) to compute RT in 12 bands from 4 to $100 \mu \mathrm{m}$. Although the original Fu and Liou correlated- $k$ distribution has known deficiencies, especially in the use of Roberts water vapor continuum (Roberts et al. 1976), it is adequate for this study because it is employed for both the MD and IPA calculations. The radiative transfer calculation includes emission, absorption, and scattering effects. Thermal IR radiative transfer tends to be dominated by emission and absorption, so the neglect of scattering is often warranted and leads to a simplified computation of thermal RT. This assumption, however, produces little advantage when using SHDOM because very few source function iterations are required for highly absorbing media.

We perform simulations of two different boundary layer cloud systems: a small cloud fraction broken trade cumulus regime and solid stratocumulus. Initial conditions and forcings for the solid stratocumulus cloud simulation are taken from the Atlantic Stratocumulus Transition Experiment (ASTEX) A209 case as simulated by Khairoutdinov and Kogan (1999). The broken trade cumulus case uses the same initial conditions and forcings as those employed in the LES intercomparison of the Barbados Oceanographic and Meteorological Experiment (BOMEX) trade cumulus case (Siebesma et al. 2003).

Cloud microphysical processes were treated as simply as possible by specifying a simple moist saturation adjustment. This precluded the production of precipitation and its associated feedbacks on the dynamical fields, which would have made the identification and interpretation of MD effects much more difficult.

The eddy-resolving model configuration for the snapshot RT calculations is a two-dimensional geometry ${ }^{3}$ $(200 \times 126$ points $)$ with horizontal and vertical grid spacings of 50 and $10 \mathrm{~m}$, respectively, in the ASTEX simulation. The BOMEX grid is $128 \times 76$ points with grid spacings of 100 and $40 \mathrm{~m}$. The purpose of the comparatively finer mesh of the ASTEX simulation is to represent better the entrainment process at cloud top.

Surface heat and moisture fluxes are $10 \mathrm{~W} \mathrm{~m}^{-2}$ (sensible heat) and $25 \mathrm{~W} \mathrm{~m}^{-2}$ (latent heat) for the ASTEX case and 9.5 and $154 \mathrm{~W} \mathrm{~m}^{-2}$, respectively, for the BOMEX simulation. Cloud optical properties are calculated from Mie theory assuming a gamma droplet size distribution with a fixed number concentration of

\footnotetext{
${ }^{3}$ We refer to our SHDOM radiative transfer calculations as multidimensional rather than three-dimensional primarily because of the use of a two-dimensional domain. Fully threedimensional radiative transfer would require a 3D spatial domain.
}

$50 \mathrm{~cm}^{-3}$. Longwave fluxes do not exhibit the strong sensitivity to cross-sectional area that forms the basis of the Twomey effect in the shortwave, so the choice of number concentration in our work is not critical. SHDOM assumes an atmospheric profile tuned to produce a downwelling IR flux at cloud top of approximately $200 \mathrm{~W} \mathrm{~m}^{-2}$. For both the ASTEX and BOMEX cases, this corresponds to a jump at cloud top of $\sim 75 \mathrm{~W} \mathrm{~m}^{-2}$.

\section{b. Interactive simulation configuration}

The ERM is coupled with SHDOM in an interactive fashion and run for both the BOMEX and ASTEX cases. Because of computational expense, all simulations are two-dimensional, and the domain is $128 \times 76$ points, the same domain size as in the snapshot simulations. Simulating buoyancy-driven flows in a twodimensional framework has a long history but is subject to some caveats, as discussed in Moeng et al. (2004). Two-dimensional simulations are generally able to represent the vertical distribution of scalar fluxes like heat and moisture, and with tuning can capture the overall boundary layer energetics, including the partitioning between resolved and unresolved fluxes. The latter includes not only the subgrid scale but also the contribution from 3D motions not resolved in the 2D model architecture (Krueger 1988).

The simulations are performed using the coupled ERM-SHDOM configuration, one with the full multidimensional treatment of RT, and the other under the IPA mode of SHDOM. The RT calculation is performed every $20 \mathrm{~s}$ rather than every time step, as is usually done in ERM and LES computations. Because the ERM domain is translated by the geostrophic wind, the evolution of the model fields is primarily due to turbulent boundary layer processes and not simply advection by the mean wind. Compared to calculating RT every time step, we estimate the RMS error introduced by the RT calculation time step to be $\sim 3 \%$ for the ASTEX simulation. This low magnitude of error makes physical sense because the calculation time step is much less than the eddy overturning time scale $(H / W \approx$ $1000 \mathrm{~s})$.

SHDOM is configured to achieve a balance between accuracy and computational expense. Accuracy is evaluated for a horizontally homogeneous cloud, for which MD and IPA fluxes should be identical. For the parameters chosen, the absolute and relative errors for the net MD flux across cloud top are $-1.5 \mathrm{~W} \mathrm{~m}^{-2}$ and $-2 \%$. These small absolute and relative errors are numerical in nature, with the most relevant parameters in dictating the accuracy of the radiative transfer calculation being the number of zenith and azimuthal angles employed, as well as the parameter that determines the 
conditions for the dynamic spatial grid adaptation [the parameter "splitacc"; see Evans (1998) for more details] used internally in SHDOM. This error is not a comparison of MD and IPA fluxes but rather is a measure of the accuracy of the calculated fluxes for the chosen SHDOM parameters, relative to a benchmark calculation with greater angular resolution and a grid adaptation parameter that ultimately results in greater spatial resolution. The error can be reduced by increasing the angular resolution or the number of dynamically added grid points (via the splitacc threshold), both at added computational expense, of course. For our simulations, we choose 8 and 16 zenith and azimuth angles, respectively, and a splitacc value of 0.5 .

Coincidentally, the difference in net domain-average flux between MD and IPA calculations employing the ASTEX and BOMEX cloud fields is similar, about $-1.5 \mathrm{~W} \mathrm{~m}^{-2}$ and $-2 \%$. Typical differences in local net fluxes between MD and IPA calculations for the ASTEX and BOMEX cloud fields range from 5 to 20 $\mathrm{W} \mathrm{m}^{-2}$ in absolute value. Therefore, the error is judged to be reasonable because the flux and heating rate errors associated with the radiation calculation are generally small relative to the local MD effects associated with cloud structure variability.

The model is run for a length of time ( $1 \mathrm{~h}$ for the solid ASTEX case, $2.58 \mathrm{~h}$ for the BOMEX case) using its own 1DRT scheme to establish a reasonable boundary layer structure that is consistent between the MD and IPA simulations. Then, simulations are continued using the coupled configuration. Our BOMEX simulation is somewhat modified from that specified in Siebesma et al. (2003). In the original, convection is forced by surface sensible and latent heat fluxes $\left(9.5\right.$ and $154 \mathrm{~W} \mathrm{~m}^{-2}$, respectively) in addition to large-scale drying and cooling terms to ensure radiative-convective equilibrium. We depart from the specifications by additionally allowing interactive radiative cooling (either MD or IPA), an approach taken by Stevens et al. (2001), who employed a simple LWP-dependent flux to add as much as $74 \mathrm{~W} \mathrm{~m}^{-2}$ of radiative cooling to the cloud. The BOMEX case was run for $12 \mathrm{~h}$ to emphasize any potential long-term MD effect on the cloud system ensemble.

\section{Broken cloud case (BOMEX)}

Figure 1 summarizes the evolution of the BOMEX simulations. Included with the interactive MD and IPA simulations is a simulation with no radiative forcing, which allows the evaluation not only of the relative effect of MD and IPA on cloud dynamics but also of the overall importance of radiative effects.
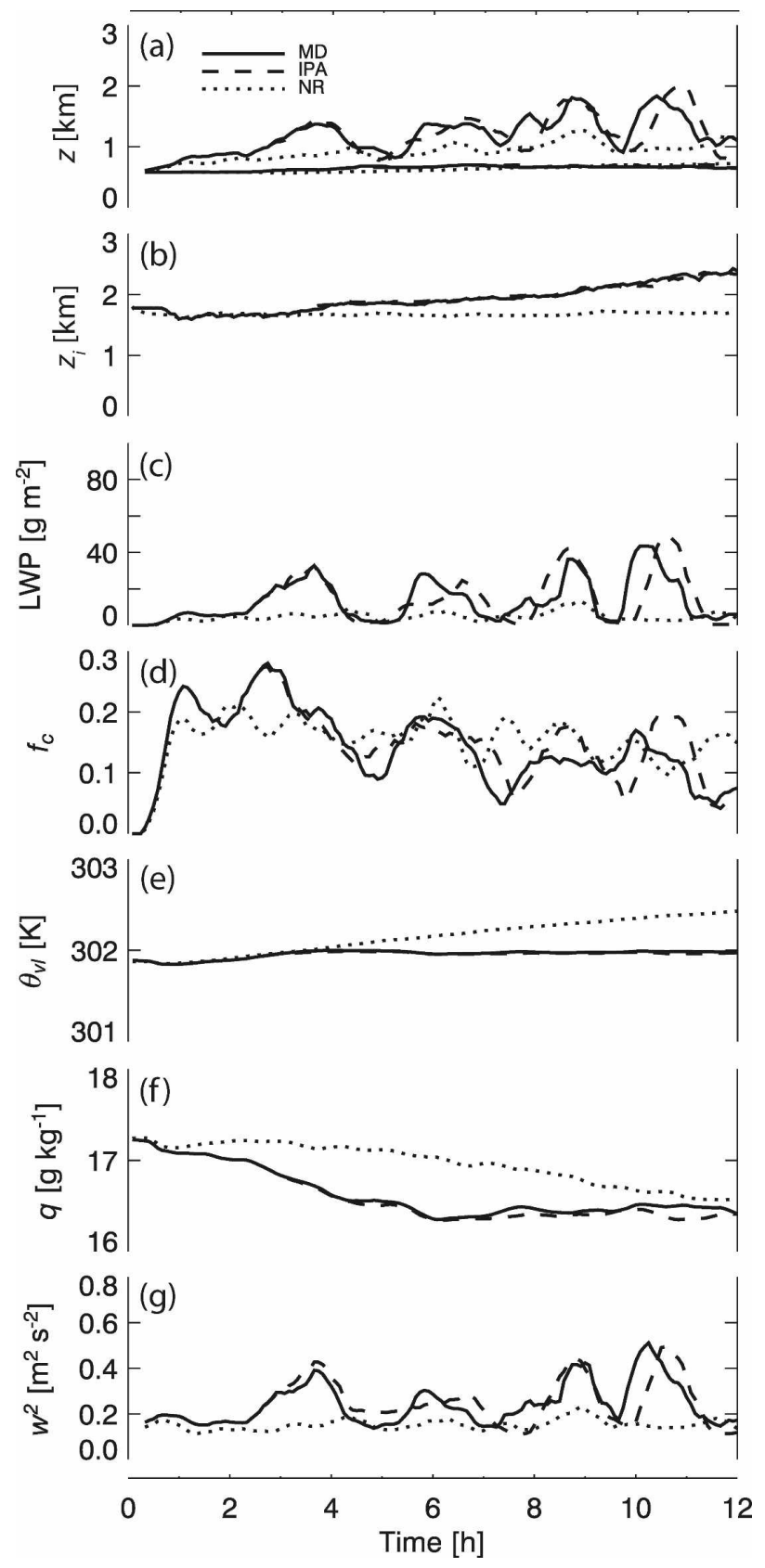

FIG. 1. Time series of ERM quantities from 1 to $12 \mathrm{~h}$ for the broken BOMEX simulations: (a) Mean cloud base and cloud top height, (b) mean inversion height, (c) LWP, (d) cloud fraction, (e) surface liquid water virtual potential temperature, (f) surface mixing ratio, and $(\mathrm{g})$ vertical velocity variance. The solid and broken lines represent the MD and IPA cases, respectively; the dotted line represents no radiative forcing.

The added forcing term from the radiation has a pronounced effect on the cloud system evolution, structure, and energetics. Without the additional radiative term, mean cloud top is consistently hundreds of meters shallower and entrainment significantly weaker relative 
to the cases employing radiation. Interactive radiation promotes deeper clouds with higher liquid water contents (higher LWP), and the surface conditions for the interactive simulations are consistently cooler and drier relative to the "no radiation" simulation, likely the result of stronger fluxes and vertical transport. The vertical velocity variance $\left\langle w^{\prime 2}\right\rangle$, a proxy for mean boundary layer turbulence intensity, is much greater in the simulations employing interactive radiation.

Differences between the MD and IPA simulations, however, are more subtle. In particular, the quantities that are more time integrated in nature (e.g., $z_{i}, \theta_{v l}, q$ ) experience little difference between MD and IPA simulations. The more instantaneous quantities exhibit noisier behavior, as expected, but even for those quantities MD and IPA statistics do not differ significantly. The only apparent difference is perhaps a slight lag in the signal for the MD case.

The spatial distribution and evolution of the MD and IPA heating rates with respect to the cloud structuresthe snapshot calculations-are shown in Fig. 2. We emphasize that the MD simulation supplies the cloud fields (as in all the snapshot analyses) on which MD and IPA radiative transfer are calculated.

This figure then represents a series of MD and IPA snapshots given a cloud field evolution. The panel series represents a "zoomed" portion of the domain shown at the top, and evolution proceeds downward from a time of $9300 \mathrm{~s}(\sim 2.58 \mathrm{~h})$, with $300 \mathrm{~s}$ separating each row. The whole series of rows represents an evolution of the cloud system structure over a 25-min period, and the period was chosen to be qualitatively representative of an individual cloud life cycle.

At first glance, the MD and IPA forcings seem remarkably similar, both in pattern and magnitude. The difference field $\left(Q_{\mathrm{md}}-Q_{\mathrm{ipa}}\right)$ indicates that MD radiative transfer tends preferentially to cool, narrow, isolated, cloudy updraft "columns" and to warm (i.e., less cooling) cloud crevices or valleys. The anomalous MD cooling in the isolated column clouds decreases significantly as the cloud entrains, reaches its equilibrium level, and takes on the characteristic mushroom thermal shape. In this case, the remaining column underlying the cap of the thermal is receiving significant radiation from the overhanging cap, as opposed to downwelling from free space in the isolated configuration, which explains the preferential warming. The difference field for the shallow scud near cloud base is predominantly positive, which is unsurprising for the same reason; it is receiving radiation from adjacent warm cloud towers rather than from space.

A statistical snapshot of the behavior of $Q_{\mathrm{md}}-Q_{\mathrm{ipa}}$ for all cloudy points over the 25 -min cloud period is presented in Fig. 3. The predominant signal is that of weak, anomalous MD cooling throughout the cloud layer. Anomalously positive regions tend to be fewer, although the spread of $Q_{\mathrm{md}}-Q_{\mathrm{ipa}}$ seems to be greater. These regions of large positive $Q_{\mathrm{md}}-Q_{\mathrm{ipa}}$ tend to be associated with crevice regions like those visible in Fig. 2. Also apparent is the concentration of weakly positive $Q_{\mathrm{md}}-Q_{\mathrm{ipa}}$ at low levels in the cloud, which represents the anomalous warming of the shallow scud.

A clearer picture of the actual cloud forcing terms is presented in Fig. 4a, which illustrates the large number of cloudy points containing weak anomalous MD cooling that happen to be associated with weak radiative forcing. Regions of stronger radiative forcing tend to be anomalously warmed, consistent with Fig. 3. Figure 4 also captures the absolute warming of a large number of cloud base points. The probability density functions (PDFs) in Fig. 4b illustrate these points (i.e., the large number of weak anomalous coolings and the small number of points anomalously warmed) most clearly.

Previous studies concluded that this MD radiative effect is largely a function of cloud geometry, specifically cloud shape and spacing. To explore this geometry effect, we calculated MD and IPA radiative transfer for a series of regularly spaced, rectangular clouds. Cloud liquid water content was defined either as a constant value of $0.5 \mathrm{~g} \mathrm{~m}^{-3}$ or as linearly increasing to that magnitude from cloud base to represent its adiabatic value. These idealized clouds crudely mimic the BOMEX clouds in Fig. 2, with both width and depth of $1 \mathrm{~km}$. Figure 5 is an example of a field of these idealized adiabatic clouds. The MD forcing in Fig. 5 exhibits cloud top cooling and weaker cooling of the cloud lateral boundaries. IPA forcing of the same cloud field (not shown) exhibits only the component of the cooling associated with the cloud top. The constant LWC clouds are accompanied by a weak cloud base warming term, which in the adiabatic cloud is distributed over a deeper layer above cloud base.

It is no surprise that both for constant and adiabatic LWC the relative contribution of the additional MD forcing to the total energetics $Q_{\text {rel }}$ is a strong function of cloud fraction $f_{c}$. Here, $Q_{\text {rel }}$ is defined as

$$
Q_{\mathrm{rel}}=\frac{\sum_{i}\left[\left(Q_{\mathrm{md}_{i}}-Q_{\mathrm{ipa}_{i}}\right) \rho_{i}\right]}{\sum_{i} Q_{\mathrm{ipa}_{i}} \rho_{i}},
$$

evaluated over each $i$ cloudy point. The radiative forcing $\left(\mathrm{K} \mathrm{h}^{-1}\right)$ is weighted by the air density $\rho_{i}$ so that $Q$ at different vertical levels may be compared on an energetically consistent basis; $Q_{\text {rel }}$ increases rapidly with decreasing $f_{c}$ until $\sim 0.85$, after which $Q_{\text {rel }}$ asymptotes 

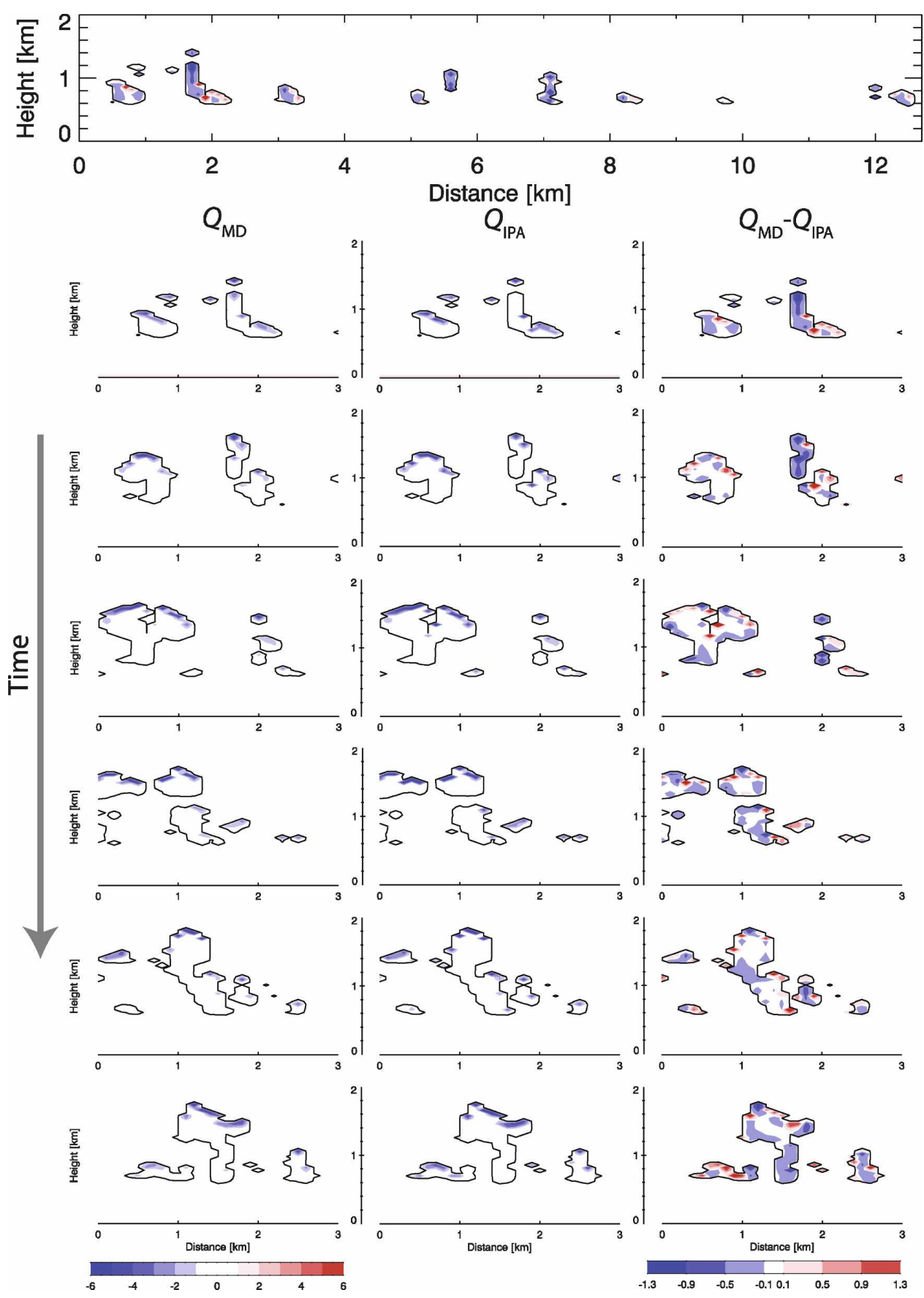

FIG. 2. The evolution of a typical BOMEX broken cloud structure and its associated radiative forcing rates $\left(\mathrm{K} \mathrm{h}^{-1}\right)$ employing (left) $\mathrm{MD}$ and (center) IPA radiative transfer. The cloud fields are obtained from the MD simulation. (right) The heating rate difference between MD and IPA rates. The small panels are from an enlarged portion of the domain from $x=0-3 \mathrm{~km}$. Each successive row represents instantaneous samples of the cloud and radiative forcing fields taken every $5 \mathrm{~min}$.

(Fig. 6) to values of 0.22 and 0.32 for the constant and adiabatic LWC cases, respectively. The IPA forcing for constant and adiabatic LWC cases is very similar, and the difference in $Q_{\text {rel }}$ is driven by the multidimensional radiative effects on the lower part of the cloud, which is characterized by smaller LWC and emissivity.

Calculating $Q_{\text {rel }}$ according to Eq. (1) for the broken cloud field in Fig. 2 results in a value of 0.056, dramati- 


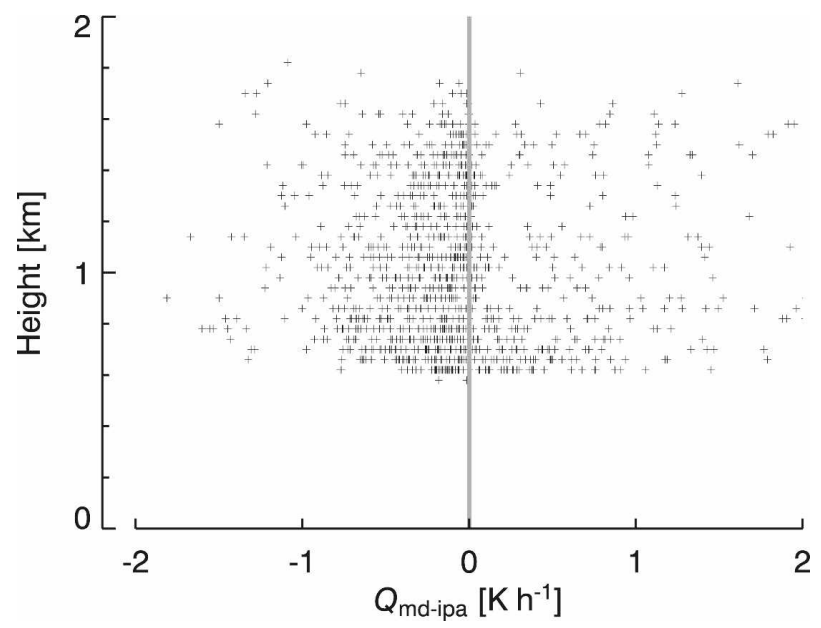

FIG. 3. Scatterplot of $Q_{\mathrm{md}}-Q_{\mathrm{ipa}}$ as a function of height for all cloudy points in the BOMEX case.

cally different from the contributions of 0.22 and 0.32 for the idealized cloud configurations. The reason for the significant difference is touched on in the earlier description of the radiative forcing cross sections. Even though cloud fraction is small in this case, in the complicated cloud structures many of the cloud lateral boundaries intercept radiation from nearby warm cloud rather than from (cold) space, so, when integrated over solid angles, the net flux is reduced relative to the isolated, rectangular cloud cases, thereby reducing the cooling. The morphology and evolution of shallow convection in nature is undoubtedly more complicated than this simple "chimney" geometry and tends to damp the magnitude of MD radiative effects.

\section{Solid cloud case (ASTEX)}

The vertical cloud structure of the unbroken case in Fig. 7 is typical of a cloud-topped boundary layer, with a roughly linear and nearly adiabatic increase with height and a relatively sharp gradient at the base of the inversion. A jump of approximately $80 \mathrm{~W} \mathrm{~m}^{-2}$ of longwave flux is present at cloud top, with a much smaller $\left(<10 \mathrm{~W} \mathrm{~m}^{-2}\right)$ jump associated with cloud base. The small cloud base jump is a result of the surface temperature being only slightly higher than that of cloud base. Associated with these flux jumps are the cooling just inside the cloud top and a slight warming at cloud base. When the $70 \mathrm{~W} \mathrm{~m}^{-2}$ of net radiative flux (80$10=70 \mathrm{~W} \mathrm{~m}^{-2}$ ) across the cloud is compared to surface sensible $\left(10 \mathrm{~W} \mathrm{~m}^{-2}\right)$ and latent $\left(25 \mathrm{~W} \mathrm{~m}^{-2}\right)$ heat fluxes, it becomes clear that this cloud is predominantly radiatively driven.

Figure $7 \mathrm{~d}$ illustrates the difference in mean radiative
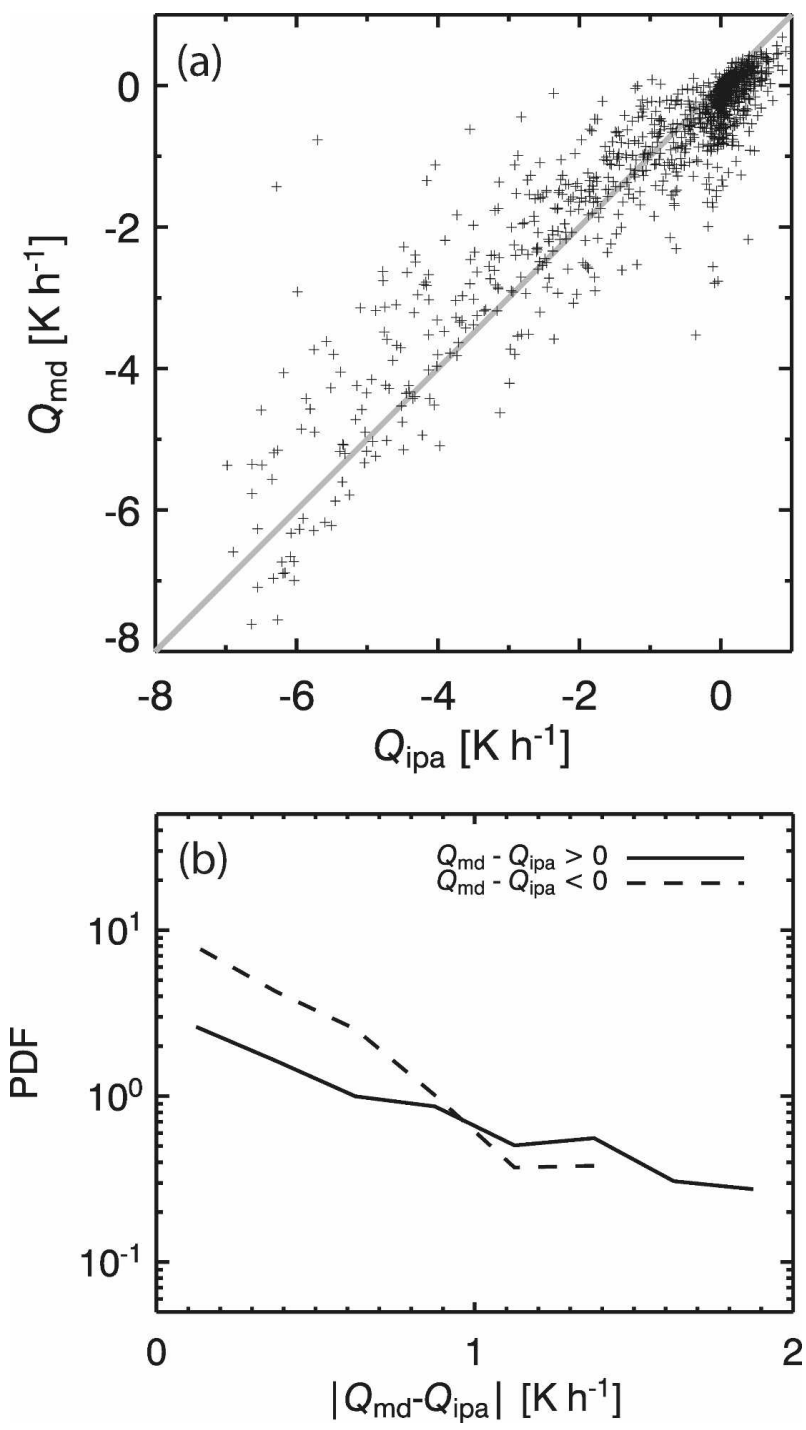

FIG. 4. Multidimensional cooling and warming in the BOMEX case, conditionally sampled on cloudy grid points ( $\mathrm{LWC} \geq 0.01 \mathrm{~g}$ $\mathrm{m}^{-3}$ ). (a) Scatterplot of $Q_{\mathrm{md}}$ and $Q_{\mathrm{ipa}}$; (b) PDFs for regions of anomalous MD warming $\left(Q_{\mathrm{md}}-Q_{\mathrm{ipa}}>0\right)$ and cooling $\left(Q_{\mathrm{md}}-\right.$ $\left.Q_{\text {ipa }}<0\right)$.

forcing between the MD and IPA cases. The net difference in forcing between MD and IPA calculations is $1.6 \%$, the positive magnitude indicating that the MD forcing (i.e., predominantly from cloud top cooling) is less than the IPA forcing. The forcing, however, is not uniformly $1.6 \%$ less everywhere; instead, regions of both enhanced and reduced forcing exist. This is apparent in Fig. 8a, particularly for magnitudes of $Q$ as high as $-6 \mathrm{~K} \mathrm{~h}^{-1}$. As for the BOMEX case, all values of $Q$ are weighed by density to be energetically comparable. For stronger forcing (more negative values of $Q$ ), the multidimensional effect tends to predominantly reduce the magnitude of $Q$. The PDFs of $Q_{\mathrm{md}}-Q_{\mathrm{ipa}}$ in $8 \mathrm{~b}$ 


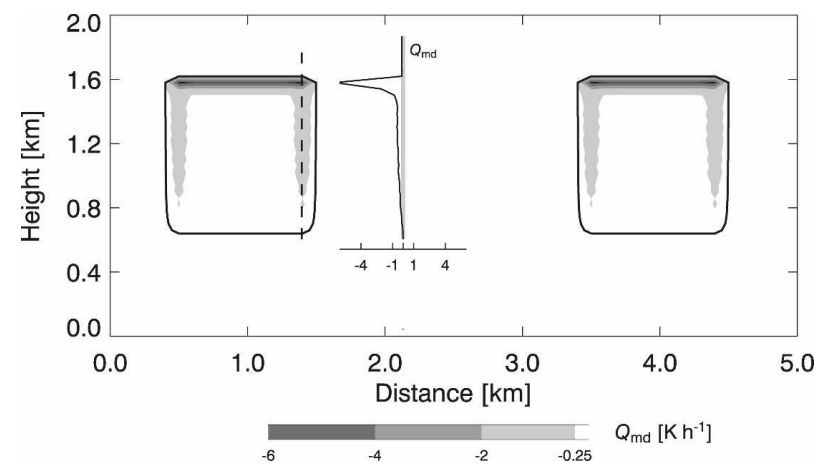

FIG. 5. MD radiative forcing for an idealized system of periodically spaced $2 \mathrm{D}$ clouds with a rectangular cross section. Solid contours represent cloud boundaries, and the vertical profile represents the radiative forcing on the side of the cloud, as indicated by the dashed vertical line.

indicate that the MD warming anomalies predominate, especially for larger values of $Q_{\mathrm{md}}-Q_{\mathrm{ipa}}$; in other words, there tends to be a greater overall amount of reduced rather than enhanced $\mathrm{MD}$ forcing.

The largest differences between MD and IPA forcing are about $1-2 \mathrm{~K} \mathrm{~h}^{-1}$; these differences, although relatively few in number, are a significant fraction of the domain-mean maximum cloud top cooling rates of about $-7 \mathrm{~K} \mathrm{~h}^{-1}$. Anomalous warming constitutes $5.1 \%$ of the total energetic contribution, whereas the anomalous cooling is $2.5 \%$, leaving a net MD effect of a $2.6 \%$ warming.

$\mathrm{MD}$ and IPA radiative transfer calculations corresponding to the cloud field at $3 \mathrm{~h}$ illustrate the complex structure of the forcing associated with the MD effects. The structure of the radiative forcing relative to the cloud water and dynamical fields exhibits a complex

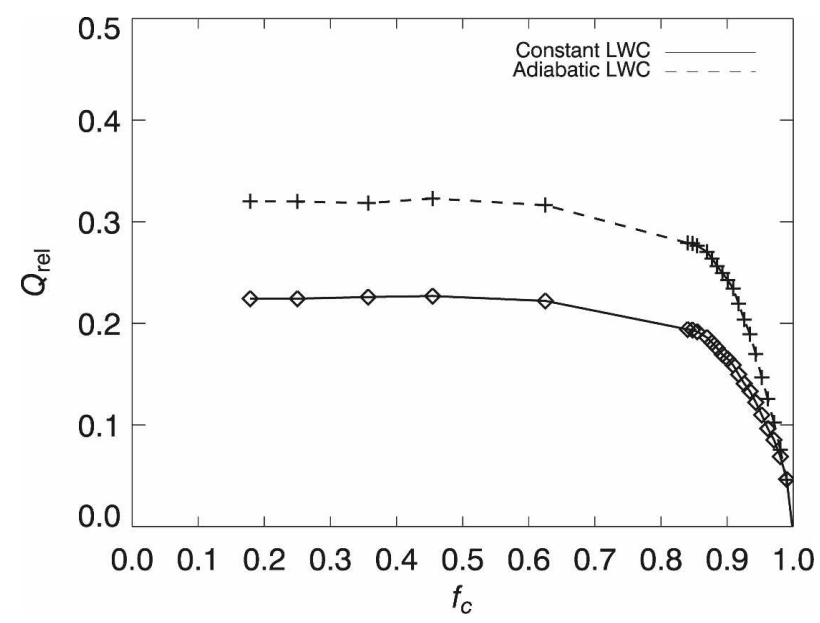

FIG. 6. Relative contribution of the additional MD forcing to the total energetics for the idealized 2D clouds in Fig. 5. relationship between $Q_{\mathrm{md}}-Q_{\mathrm{ipa}}$ and variations in cloud top (Fig. 9). Internal cloud LWC variability is also associated with variability in $Q_{\mathrm{md}}-Q_{\mathrm{ipa}}$, although to a lesser extent. Regions of both enhanced and reduced forcing exist, mainly within the top $50 \mathrm{~m}$ or so of the cloud. For this unbroken cloud system, the differences between MD and IPA radiative forcing rates are most prominent near cloud top undulations. Anomalous cooling $\left(Q_{\mathrm{md}}-Q_{\text {ipa }}<0\right)$ is generally associated with regions of high cloud tops, whereas anomalous warming $\left(Q_{\mathrm{md}}-Q_{\mathrm{ipa}}>0\right)$ is associated with low cloud tops. Closer inspection of Fig. $9 \mathrm{~b}$ reveals the existence of multiple scales of cloud top undulations and a relationship between the forcing anomalies and the concavity of the cloud top. The shape of the undulating cloud top is largely dictated by the eddy structure of updrafts and downdrafts, with billows (high cloud tops) associated with updrafts and valleys or crevices (low cloud tops) associated with downdrafts. The PDF in Fig. 8b indicates that the reduced forcing in the cloud top crevices is more prominent than enhanced forcing in the cloud top billow regions.

It is relevant to mention that increasing the spatial resolution of the ERM would better represent the entrainment process and lead to greater variability in cloud top structure. But how fine must the grid spacing be to resolve these processes completely? Observations by Gerber et al. (2005) show cloud top regions of depleted liquid water as narrow as $5 \mathrm{~m}$ in size. Clearly, we are not representing this scale of variability in our ERM, but relative to our results we can speculate that the greater variability would lead to larger magnitudes of local anomalous warming and cooling, but likely with little change in the mean forcing.

Because the mean radiative forcing is quite small because of the canceling effect of positive and negative anomalies, we might be tempted to conclude that any MD effect would be insignificant. However, in Figs. $9 \mathrm{a}, \mathrm{b}$ it is apparent that the regions of anomalous cooling are generally associated with cloud top billows that typically accompany boundary layer updrafts. Similarly, anomalous warming is present in cloud top valleys, which are normally in downdraft regions. This idea is cast in Fig. 9c as the covariation between the vertical velocity and the difference between MD and IPA forcing: ${ }^{4} w^{\prime}\left(Q_{\mathrm{md}}-Q_{\text {ipa }}\right)$. Figure $9 \mathrm{c}$ contains regions of positive and negative covariation, but the mean profile of covariance $\overline{w^{\prime}\left(Q_{\mathrm{md}}-Q_{\mathrm{ipa}}\right)}$ in Fig. 10 shows that the cloud top variability scale that most closely matches the

\footnotetext{
${ }^{4}$ We distinguish here between the local (fluctuating) covariation event and its average value covariance, which is overlined.
} 

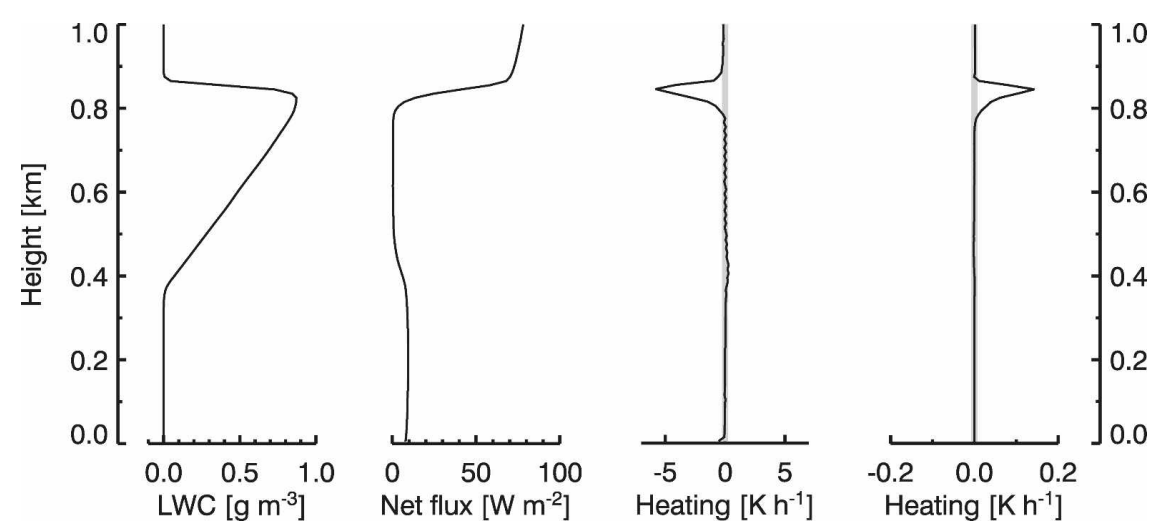

FIG. 7. Mean profiles from the radiative transfer calculations performed on the MD ASTEX cloud fields. From left to right: LWC, net MD longwave flux, MD heating rate (from net MD flux divergence), and the difference between MD and IPA heating rates.

boundary layer eddy structures tends to be reliably associated with negative values of covariation. Although this quantity does not rigorously correspond in a Reynolds averaging sense to any term in the governing equations, it can be thought of as being qualitatively related to a difference in buoyancy flux between MD and IPA simulations, which in the simulations would be exhibited as different turbulent kinetic energy (TKE) buoyancy source terms. Physically, notions of thermal buoyancy dictate that this negative correlation will tend to damp the PBL energetics.

The weakly positive $\overline{w^{\prime}\left(Q_{\mathrm{md}}-Q_{\mathrm{ipa}}\right)}$ at cloud base in Fig. 10 may be understood using similar reasoning. Like cloud top, cloud base is characterized by upward and downward undulations. In the MD case, cloud base in upward undulating regions receives contributions of longwave radiation not only from the warm surface, but also from the cooler surrounding cloud. In the IPA case, all the radiation comes from the warm surface. Unlike for cloud top, cloud base updrafts are regions of low cloud base, and downdrafts are regions of elevated cloud base. This is a result of the asymmetry of the stratocumulus circulation, especially when associated with strong entrainment or drizzle effects (Stevens et al. 1998). Having updraft associated with anomalous warming and downdraft associated with anomalous cooling results in the weak positive covariance at cloud base.

In the fully interactive simulations, the longwave MD effect is realized via the manner in which the MD heating rates modify the thermal buoyancy field relative to the IPA solution. This mechanism is the direct radiative-dynamic feedback of interest. Because the ERM is run for $2 \mathrm{~h}$ using its own two-stream RT scheme to establish a reasonable boundary layer structure, both simulations are identical until then. For the next $4 \mathrm{~h}$, the two simulations employ MD and IPA heating rates obtained from SHDOM.

Throughout the simulation, the MD radiative forcing is systematically weaker, by $1.5 \mathrm{~W} \mathrm{~m}^{-2}$, than the IPA forcing. Thus, the spatial relationship between the forcing anomaly and the dynamics does not only imply a damping of the dynamics; weaker forcing should result in weaker dynamical structures. The difference between mean MD and IPA forcing is robust over the 4-h course of the simulation.

Figure 11 summarizes the evolution of mean boundary layer statistics for the solid cloud case. Differences between MD and IPA simulations develop but appear to show little in the way of systematic bias in this relatively short $(6 \mathrm{~h})$ simulation. The initial differences in turbulent kinetic energy (TKE; Fig. 11f) that develop between simulations at $2.5 \mathrm{~h}$ are consistent with the reduced radiative forcing in the MD case. However, the IPA TKE quickly drops below that of the MD simulation. The buoyancy flux behaves similarly (Fig. 11e). The noisiness in these fields is unsurprising, especially given the small domain, and is tied to particular manifestations of the dynamical field. Long time averages should, however, reveal systematic differences. Similarly, process-integrated quantities such as $z_{i}$, which evolve over many dynamical configurations, should exhibit systematic behavior. In fact, the MD simulation does exhibit a very slightly reduced entrainment, as evinced by the slight divergence of $z_{i}$ solutions in Fig. 11a, although this difference is not likely to be statistically (or dynamically) significant in view of the spacetime variability.

\section{Discussion and conclusions}

At first thought, solid stratocumulus clouds would seem to be the ideal application for IPA radiative trans- 

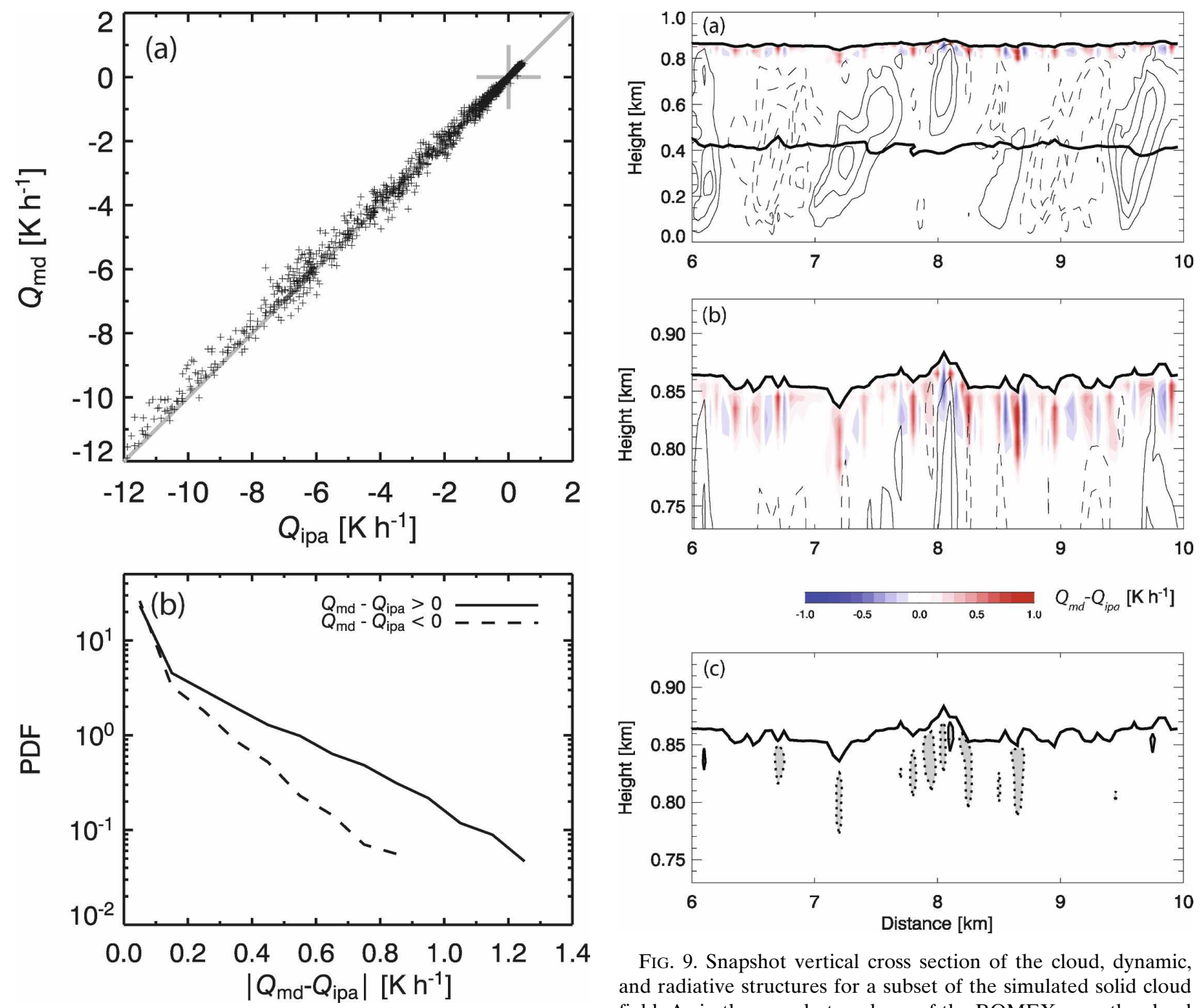

FIG. 8. Multidimensional cooling and warming of cloudy regions for the unbroken ASTEX case. (a) Scatterplot of $Q_{\mathrm{md}}$ and $Q_{\mathrm{ipa}}$; (b) PDFs for regions of anomalous MD warming $\left(Q_{\mathrm{md}}-Q_{\text {ipa }}>\right.$ $0)$ and cooling $\left(Q_{\mathrm{md}}-Q_{\mathrm{ipa}}<0\right)$.

fer. Closer inspection of our ASTEX case study reveals cloud top undulations and internal LWC variability that can introduce multidimensional effects in the radiative forcing that only slightly reduce the overall cloud top cooling (forcing) but can significantly redistribute that forcing, generally enhancing it in upwardly perturbed billow regions and reducing it in downwardly perturbed valley or crevice regions. The correlation between these radiative forcing anomalies and the cloud dynamical structures implies a negative feedback that should damp the boundary layer dynamics. Because of the chaotic nature of turbulent flows, the interactive simulations develop significant differences in individual boundary layer eddy structures. However, the MD and

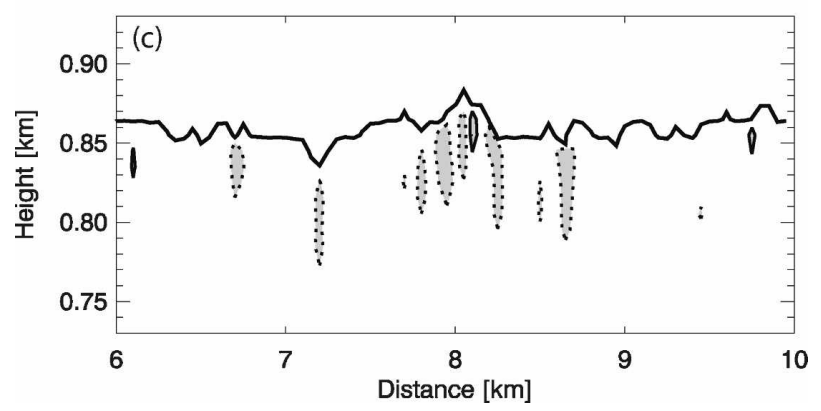

FIG. 9. Snapshot vertical cross section of the cloud, dynamic, and radiative structures for a subset of the simulated solid cloud field. As in the snapshot analyses of the BOMEX case, the cloud fields here are obtained from the MD simulations. (a) Vertical velocity (contour interval of $0.5 \mathrm{~m} \mathrm{~s}^{-1}$; negative values dashed) and radiative forcing anomaly $Q_{\mathrm{md}}-Q_{\mathrm{ipa}}$ (colors). Bold lines represent cloud boundaries. (b) Zoomed portion of the top hundred meters of the cloud. (c) Covariation between dynamics and forcing anomaly $w^{\prime}\left(Q_{\mathrm{md}}-Q_{\mathrm{ipa}}\right)$. Gray areas are regions where $\left|w^{\prime}\left(Q_{\mathrm{md}}-Q_{\text {ipa }}\right)\right| \geq 3 \times 10^{-5} \mathrm{~m} \mathrm{~K} \mathrm{~s}^{-2}$, with dashed and solid contours surrounding negative and positive regions, respectively.

IPA solutions exhibit very little difference in "cumulative" quantities like the height of the inversion $z_{i}$ or mean turbulence intensity $\left\langle w^{\prime 2}\right\rangle$. A slightly smaller entrainment rate in the MD simulation is consistent both with the reduced forcing $(\sim 2.5 \%)$ and with the negative correlation of the anomalies and the perturbation structures. Our results indicate that the common assumption of IPA, specifically applied to employing 1D thermal radiative transfer in cloud models, appears to be reasonable.

The broken BOMEX case would appear to be much 


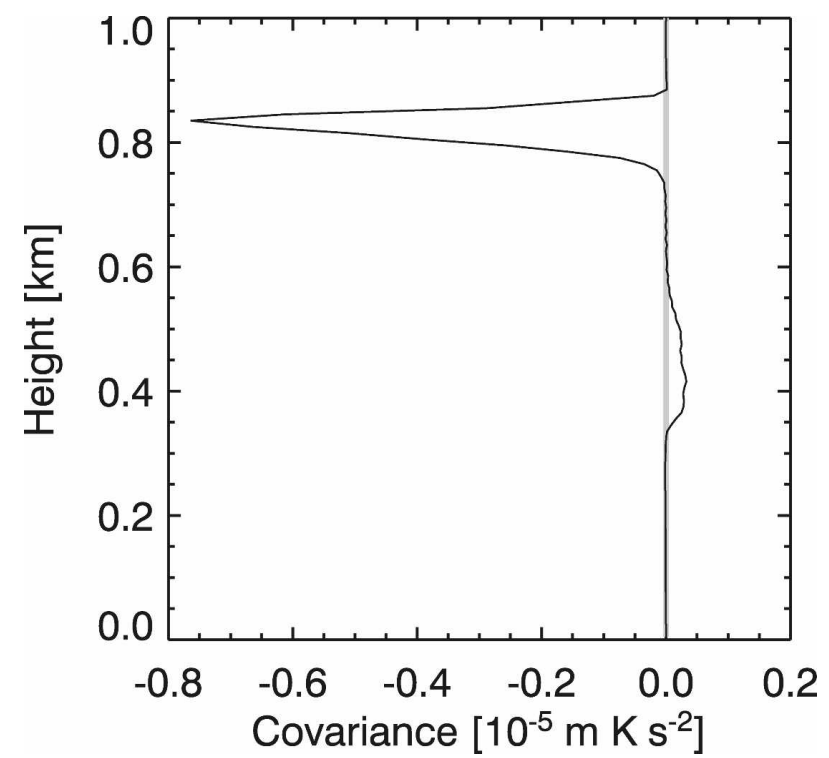

FIG. 10. Vertical profile of $\overline{w^{\prime}\left(Q_{\mathrm{md}}-Q_{\mathrm{ipa}}\right)}$ for the unbroken ASTEX cloud system averaged horizontally over the realization in Fig. 9.

more supportive of potential MD effects. We thought lateral cooling of isolated towers might play a role in modifying the cloud dynamics, as was shown previously by Guan et al. (1997) for a simulated isolated cumulus cloud. Radiative transfer calculations for idealized rectangular clouds indicate contributions to the radiative forcing from the horizontal flux of as much as $32 \%$. However, for a more realistic cloud field obtained from the ERM, the horizontal flux contribution is only $5.6 \%$. The reason for this difference lies in the fact that the MD forcing includes not only the lateral cooling of cloud columns (like the idealized calculation), but also anomalous warming associated with detrained mushroom cap-shaped structures, entrainment-driven structures on cloud sides and top, and low-level scud. Each of these features contributes anomalous MD warming that tends to compete with the cooling associated with upright, isolated vertical columns.

Differences between MD and IPA simulations for the BOMEX case are even less apparent than in the plane-parallel solid cloud case, especially when compared with the simulation with no radiation whatsoever. Although differences between the simulations develop, they appear to be little more than simple time lags (e.g., Figure 1c), and cumulative quantities like $z_{i}$ are unchanged.

We have two explanations for the insensitivity of the cloud properties to MD effects: one speculative about the nature of radiative-dynamic interactions, and the other based on relative energetics of the radiative processes.
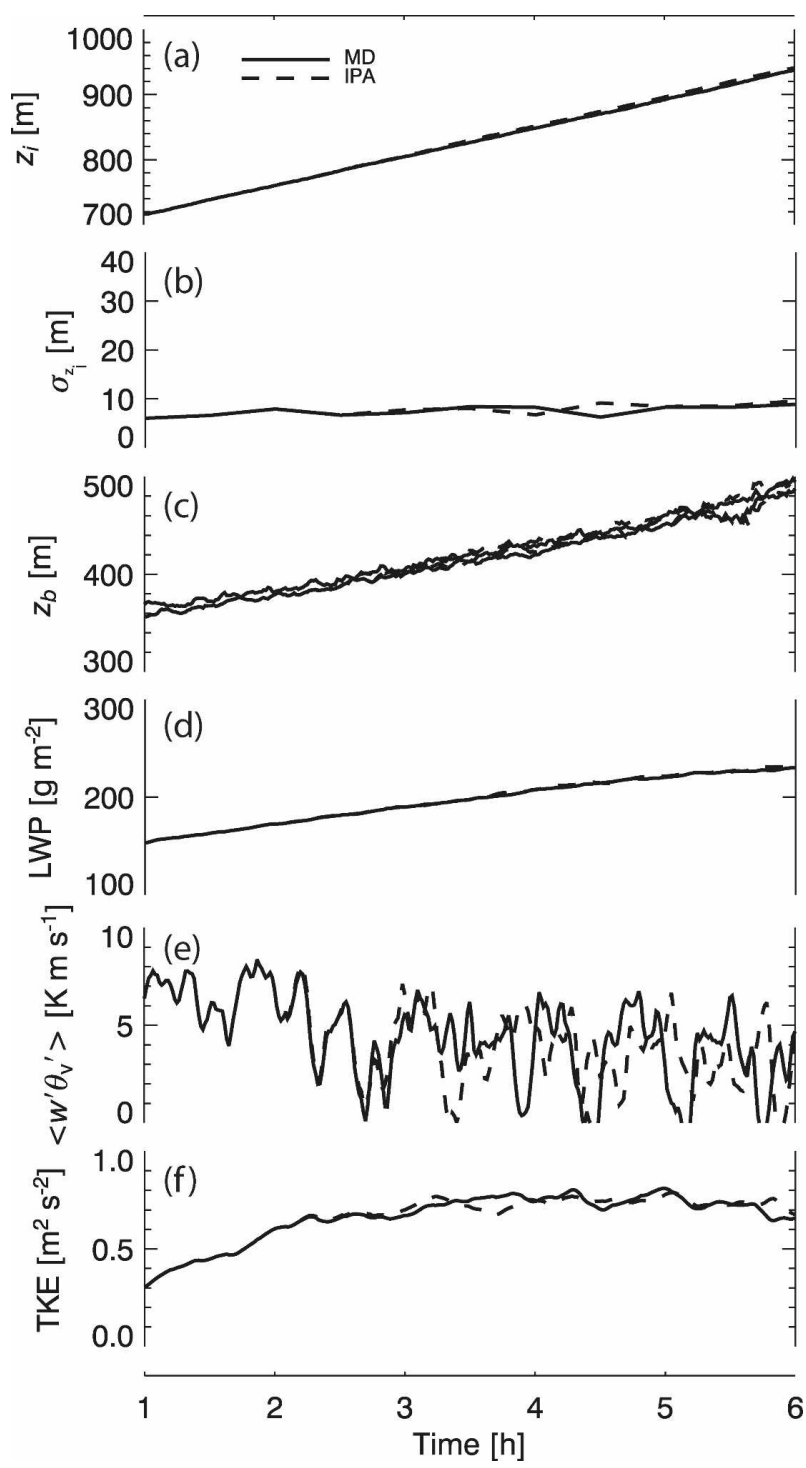

FIG. 11. Time series of ERM quantities from 1-6 $\mathrm{h}$ for the unbroken cloud simulation: (a) mean inversion height, (b) standard deviation of inversion height, (c) cloud base height for updrafts and downdrafts (updraft base is typically the lower of the two), (d) LWP, (e) resolved mean buoyancy flux over the PBL, and (f) turbulent kinetic energy.

Our results for the solid ASTEX cloud imply that the interactive relationship between radiation and dynamics is quite "loose" in the sense that the overall magnitude of the radiative forcing is far more important than particular details of the spatial distribution. This notion is in some ways consistent with the simplified treatments of longwave radiation used in recent LES model intercomparison studies (e.g., Stevens et al. 2005). Although these simplified radiation schemes do respond to changes in cloud structure, the flux is an approximation based on cloud LWP rather than directly on cloud 
radiative properties. As such, these schemes are mostly a vehicle for supplying a specified amount of radiative forcing to the cloud structure, with little focus on getting fine details of the forcing absolutely correct.

It is difficult to conceive of a simple underlying physical interpretation for this insensitivity to the spatial distribution of the forcing. The most reasonable explanation centers on the residence time of parcels in the active region of radiative cooling at cloud top. Kogan (2006) found that air parcels reside in the upper $50 \mathrm{~m}$ of the cloud layer for only minutes at a time, and even less in the upper $10 \mathrm{~m}$ of the cloud. Most parcels passing through these regions of cooling near cloud top exhibit some component of horizontal motion, and from the complicated pattern of anomalous MD forcing in Fig. $9 \mathrm{~b}$, it is very likely that any given parcel will not be confined to a single region of enhanced or reduced forcing. Rather, a parcel may pass rather quickly through regions of greater forcing, as well as through regions of less. For this reason, we suspect that the reduced mean forcing is driving the very weak entrainment rate reduction rather than the redistribution of forcing. Thus, parcels tend to feel the net MD effect (the small reduction of net forcing) rather than the particulars of the spatial distribution.

Although Fig. 1 demonstrates the importance of including longwave radiation as a whole, the small MD effects in the broken BOMEX case are probably related to the relatively small contribution of the radiative forcing to the overall energetics and of course also to the fact that the MD and IPA forcings differ by only $\sim 5.6 \%$. In the BOMEX case, the radiative forcing amounts to only about $16 \mathrm{~W} \mathrm{~m}^{-2}$. When divided by an average cloud fraction of 0.2 (see Fig. 1d), the radiative forcing over cloudy regions is $78 \mathrm{~W} \mathrm{~m} \mathrm{~m}^{-2}$, which is roughly consistent with the simplified radiative forcing term in Stevens et al. (2001). The domain-average forcing of $16 \mathrm{~W} \mathrm{~m}^{-2}$, however, is small compared to the latent heat flux $\left(154 \mathrm{~W} \mathrm{~m}^{-2}\right)$ and the large-scale temperature advection $\left(35 \mathrm{~W} \mathrm{~m}^{-2}\right)$. And, of course, $5.6 \%$ of $16 \mathrm{~W} \mathrm{~m}^{-2}$, which amounts to the difference in forcing arising from MD effects, is dwarfed by these larger terms and also by smaller terms like sensible heat flux $\left(9.5 \mathrm{~W} \mathrm{~m}^{-2}\right)$.

MD radiative transfer effects may be associated with deep convective clouds, too, although the dynamics in those clouds are most typically dictated by convective available potential energy (CAPE), as manifested by the deep, buoyant ascent of parcels originating in the boundary layer, with radiative effects important in preferential heating and cooling of the boundary layer. Our simulations of solid and broken low clouds demonstrate only the most modest of differences when comparing forcing by MD and IPA longwave radiative transfer. We do not wish to overgeneralize these results, which we believe apply specifically to how multidimensional radiative effects in the longwave influence the cloud dynamics directly.

We should also acknowledge that employing a full explicit radiative transfer method like SHDOM is in many ways a brute force approach, computationally expensive and perhaps overly complicated for a radiative transfer problem so dominated by thermal emission and extinction. By way of comparison, the MD ASTEX simulation takes approximately 250 times longer to run than when using a typical two-stream longwave treatment of radiative transfer. Could these longwave radiative transfer physics be parameterized, for example, by some form of perturbation scheme (Polonsky et al. 2003)? Perhaps, but we desired some preliminary estimate of the importance of this MD effect before making the commitment to developing a new, computationally efficient parameterization of MD radiative transfer.

These results do not provide evidence of a sufficient multidimensional effect on the cloud dynamics themselves to warrant the use of a fully interactive multidimensional radiative transfer scheme. Or perhaps more properly put, the errors arising from the neglect of MD effects in evaluating radiative forcing are smaller than other errors intrinsic in typical LES simulations (such as the subgrid-scale parameterization or model numerics). For example, the range of entrainment rates among state-of-the-art LES models is $\sim 20 \%-30 \%$ (Fig. 7 in Stevens et al. 2005), which is much larger than the subtle MD effects present in our results. Thus, it appears that the performance of current LES models is not hampered by neglecting longwave MD radiative effects and that at least for the present, employing MD longwave radiative transfer does little to enhance simulation fidelity over traditional IPA radiative transfer, as implemented in 1D two- or four-stream methods.

This study obviously has limitations, the most apparent one being tied to the two-dimensionality of the simulations. Because scattering is minimal in the longwave, the effect of MDRT can be thought of as the ratio of horizontal to vertical net radiative fluxes, which in turn depends on the geometry of the simulated clouds, in particular the ratio of cloud lateral and cloud top areas. Assuming the width and height of an idealized cloud are $2 R$ and $H$, the ratio of lateral to vertical radiative fluxes in the case of $2 \mathrm{D}$ clouds is proportional to $H / 2 R$. However, for a $3 \mathrm{D}$ cylindrical cloud this ratio is $2 \pi R H / \pi R^{2}=2 H / R$ - that is, 4 times the corresponding value for the $2 \mathrm{D}$ case. Relative to the $2 \mathrm{D}$ configuration, we might thus expect the contribution of horizontal 
fluxes in $3 \mathrm{D}$ simulations to be larger because of the larger lateral surface of 3D clouds. As a result, the relative contribution of the additional MD forcing to the total energetics $Q_{\text {rel }}$ may be much larger than the estimated value of $5.6 \%$ in our 2D BOMEX cumulus simulation, possibly as much as $\sim 20 \%$. Similarly, the effect of the warming/cooling anomalies in stratocumulus cloud top undulations may also be stronger in the $3 \mathrm{D}$ case.

Another limitation is related to the neglect of drizzle in our current simulations. Drizzle falling below cloud base is radiatively active, though undoubtedly less so than the cloud itself, because it has much lower water content distributed over larger droplets. When drizzle is present, however, it may increase the lateral surface area of cloud, leading to a larger relative contribution from lateral fluxes.

Lastly, our simulations show that the differences, however small, seem to increase with time. We might expect that for longer simulations, beyond the $12 \mathrm{~h}$ considered in our experiments, the biases may accumulate and ultimately result in a more pronounced effect on cloud parameters. Of course, beyond $12 \mathrm{~h}$ of longwaveonly radiation, the shortwave spectrum needs consideration at most latitudes and seasons. In multidimensional radiative transfer with SHDOM, the solar problem is an order of magnitude more expensive, owing to strong scattering (hence long-range radiation transport) and localized sources. The effect of multidimensional versus IPA radiative transfer on cloud dynamics may also be more significant than found here.

Further studies will be needed to quantify the impact of these limitations on cloud dynamics. Future efforts should also explore the possibility of multidimensional effects for other radiatively driven cloud systems. We previously mentioned altocumulus, which are predominantly driven by radiative forcing, but recent studies of cirrus cloud inhomogeneity (Fu et al. 2000) and horizontal photon transport in contrails (Gounou and Hogan 2007) suggest multidimensional effects may play a role in cloud dynamics for these radiatively driven cloud systems as well.

Acknowledgments. We wish to acknowledge Bob Cahalan and Ezra Takara for their early encouragement in this effort. We greatly appreciate constructive suggestions from Steve Krueger and an anonymous reviewer. This research was supported by the Office of Science (BER), U.S. Department of Energy, Grant DE-FG0205ER64062, as part of the Atmospheric Radiation Measurement Program; by ONR grants N00014-05-10550 and N00014-03-1-0304; and by NOAA/Office of Oceanic and Atmospheric Research under NOAA-
University of Oklahoma Cooperative Agreement NA17RJ1227, U.S. Department of Commerce. Some of the computing for this project was performed at the OU Supercomputing Center for Education and Research (OSCER) at the University of Oklahoma.

\section{REFERENCES}

Benassi, A., F. Szczap, A. Davis, M. Masbou, C. Cornet, and P. Bleuyard, 2004: Thermal radiative fluxes through inhomogeneous cloud fields: A sensitivity study using a new stochastic cloud generator. Atmos. Res., 72, 291-315.

Cahalan, R. F., W. Ridgway, W. J. Wiscombe, and T. L. Bell, 1994a: The albedo of fractal stratocumulus clouds. J. Atmos. Sci., 51, 2434-2455.

$-, \ldots,-$, S. Golmer, and Harshvardhan, 1994b: Independent pixel and Monte Carlo estimates of stratocumulus albedo. J. Atmos. Sci., 51, 3776-3790.

— advanced radiative transfer tools for cloudy atmospheres. Bull. Amer. Meteor. Soc., 86, 1275-1293.

Davis, A., A. Marshak, R. Cahalan, and W. Wiscombe, 1997: The Landsat scale break in stratocumulus as a three-dimensional radiative transfer effect: Implications for cloud remote sensing. J. Atmos. Sci., 54, 241-260.

Di Giuseppe, F., and A. M. Tompkins, 2003: Effect of spatial organization on solar radiative transfer in three-dimensional idealized stratocumulus cloud fields. J. Atmos. Sci., 60, 17741794.

Ellingson, R. G., 1982: On the effects of cumulus dimensions on longwave irradiance and heating rates calculations. J. Atmos. Sci., 39, 886-896.

- and E. Takara, 2005: Longwave radiative transfer in inhomogeneous cloud layers. 3D Radiative Transfer in Cloudy Atmospheres, A. Marshak and A. Davis, Eds., SpringerVerlag, 487-519.

Evans, K.F., 1998: The spherical harmonics discrete ordinate method for three-dimensional atmospheric radiative transfer. J. Atmos. Sci., 55, 429-446.

Fu, Q., and K. N. Liou, 1992: On the correlated $k$-distribution method for radiative transfer in nonhomogeneous atmospheres. J. Atmos. Sci., 49, 2139-2156.

_- B. Carlin, and G. Mace, 2000: Cirrus horizontal inhomogeneity and OLR bias. Geophys. Res. Lett., 27 (20), 3341-3344.

Gerber, H., G. Frick, S. P. Malinowski, J.-L. Brenguier, and F. Burnet, 2005: Holes and entrainment in stratocumulus. J. Atmos. Sci., 62, 443-459.

Gounou, A., and R. J. Hogan, 2007: A sensitivity study of the effect of horizontal photon transport on the radiative forcing of contrails. J. Atmos. Sci., 64, 1706-1716.

Guan, H., R. Davies, and M. K. Yau, 1995: Longwave radiative cooling rates in axially symmetric clouds. J. Geophys. Res., 100, 3213-3220.

- M. K. Yau, and R. Davies, 1997: The effects of longwave radiation in a small cumulus cloud. J. Atmos. Sci., 54, 22012214.

Harshvardhan, and J. A. Weinman, 1982: Infrared radiative transfer through a regular array of cuboidal clouds. J. Atmos. Sci., 39, 431-439.

, and R. W. L. Thomas, 1984: Solar reflection from interacting and shadowing cloud elements. J. Geophys. Res., 89, 71797185 . 
Houghton, J., Y. Ding, D. J. Griggs, M. Noguer, P. J. van der Linden, X. Dai, K. Maskell, and C. A. Johnson, Eds., 2001: Climate Change 2001: The Scientific Basis. Cambridge University Press, $881 \mathrm{pp}$.

Khairoutdinov, M. F., and Y. L. Kogan, 1999: A large eddy simulation model with explicit microphysics: Validation against aircraft observations of a stratocumulus-topped boundary layer. J. Atmos. Sci., 56, 2115-2131.

Killen, R. M., and R. G. Ellingson, 1994: The effect of shape and spatial distribution of cumulus clouds on longwave irradiance. J. Atmos. Sci., 51, 2123-2136.

Kogan, Y. L., 2006: Large-eddy simulation of air parcels in stratocumulus clouds: Time scales and spatial variability. J. Atmos. Sci., 63, 952-967.

, M. P. Khairoutdinov, D. K. Lilly, Z. N. Kogan, and Q. Liu, 1995: Modeling of stratocumulus cloud layers in a large eddy simulation model with explicit microphysics. J. Atmos. Sci., 52, 2923-2940.

Koračin, D., V. Isakov, and L. Mendez-Nuñez, 1998: A cloudresolving model with the radiation scheme based on the Monte Carlo method. Atmos. Res., 47, 437-459.

Krueger, S. K., 1988: Numerical simulation of tropical cumulus clouds and their interaction with the subcloud layer. $J$. Atmos. Sci., 45, 2221-2250.

Liu, S., and S. K. Krueger, 1998: Numerical simulations of altocumulus using a cloud resolving model and a mixed layer model. Atmos. Res., 47, 461-474.

Masunaga, H., and T. Nakajima, 2001: The effective cloud fraction of broken clouds obtained by multistream radiative transfer: Part 1. Longwave radiation. J. Atmos. Sci., 58, 2455-2467.

Mishchenko, M., 2008: Multiple scattering, radiative transfer, and weak localization in discrete random media: Unified microphysical approach. Rev. Geophys., 46, RG2003, doi:10.1029/ 2007RG000230.

Moeng, C.-H., 2000: Entrainment rate, cloud fraction, and liquid water path of PBL stratocumulus clouds. J. Atmos. Sci., 57, 3627-3643.

- J. C. McWilliams, R. Rotunno, P. P. Sullivan, and J. Weil, 2004: Investigating 2D modeling of atmospheric convection in the PBL. J. Atmos. Sci., 61, 889-903.

Polonsky, I., M. Box, and A. B. Davis, 2003: Radiative transfer through inhomogeneous turbid media: Implementation of the adjoint perturbation approach at the first-order. J. Quant. Spectrosc. Radiat. Transfer, 78, 85-98.

Roberts, R. E., J. E. A. Selby, and L. M. Biberman, 1976: Infrared continuum absorption by atmospheric water vapor in the 8-12 $\mu \mathrm{m}$ window. Appl. Opt., 15, 2085-2090.

Siebesma, A. P., and Coauthors, 2003: A large-eddy simulation intercomparison study of shallow cumulus convection. J. Atmos. Sci., 60, 1201-1219.

Siems, S. T., C. S. Bretherton, M. B. Baker, S. S. Shy, and R. E. Breidenthal, 1990: Buoyancy reversal and cloud-top entrainment instability. Quart. J. Roy. Meteor. Soc., 116, 705-739.

Stevens, B., W. R. Cotton, G. Feingold, and C.-H. Moeng, 1998: Large-eddy simulations of strongly precipitating, shallow, stratocumulus-topped boundary layers. J. Atmos. Sci., 55, $3616-3638$.

_ , and Coauthors, 2001: Simulations of trade wind cumuli under a strong inversion. J. Atmos. Sci., 58, 1870-1891.

—, and Coauthors, 2005: Evaluation of large-eddy simulations via observations of nocturnal marine stratocumulus. Mon. Wea. Rev., 133, 1443-1462.

Takara, E. E., and R. G. Ellingson, 1996: Scattering effects on longwave fluxes in broken cloud fields. J. Atmos. Sci., 53, 1464-1476.

Welch, R. M., and B. A. Wielicki, 1984: Stratocumulus cloud field reflected fluxes: The effect of cloud shape. J. Atmos. Sci., 41, $3085-3103$.

Zuidema, P., and K. F. Evans, 1998: On the validity of the independent pixel approximation for boundary layer clouds observed during ASTEX. J. Geophys. Res., 103, 6059-6074. 